\title{
Error Estimates for Filtered Back Projection
}

\author{
Matthias Beckmann and Armin Iske \\ Department of Mathematics, University of Hamburg, Germany \\ \{matthias.beckmann,armin.iske\}@uni-hamburg.de
}

\begin{abstract}
Computerized tomography allows us to reconstruct a bivariate function from its Radon samples. The reconstruction is based on the filtered back projection (FBP) formula, which gives an analytical inversion of the Radon transform. The FBP formula, however, is numerically unstable. Therefore, suitable low-pass filters of finite bandwidth are employed to make the reconstruction by FBP less sensitive to noise.

The objective of this paper is to analyse the reconstruction error occurring due to the use of a low-pass filter. To this end, we prove $L^{2}$ error estimates on Sobolev spaces of fractional order. The obtained error estimates are affine-linear with respect to the distance between the filter's window function and the constant function 1 in the $L^{\infty}$-norm. Our theoretical results are supported by numerical simulations, where in particular the predicted affine-linear behaviour of the error is observed.
\end{abstract}

\section{INTRODUCTION}

Filtered back projection (FBP) refers to a well-known and commonly used reconstruction technique from computerized tomography (CT), which deals with the generation of medical images from measurements of X-ray scans. The X-ray data can be interpreted as a finite set of line integrals for the (unknown) attenuation function which describes the amount of energy being absorbed by the scanned medium. For recovering the interior structure of the scanned object from given X-ray scans, this requires the reconstruction of the attenuation function. We formulate this basic reconstruction problem as follows.

Problem 1 (Basic reconstruction problem): For a bounded domain $\Omega \subset \mathbb{R}^{2}$, reconstruct a bivariate function $f \equiv f(x, y)$ with compact support contained in $\Omega$ from its line integrals

$$
\int_{\ell} f(x, y) \mathrm{d} x \mathrm{~d} y
$$

for all straight lines $\ell \subset \mathbb{R}^{2}$ passing through $\Omega$.

Let $\ell_{t, \theta}$ denote the unique straight line which passes through the point $(t \cos (\theta), t \sin (\theta)) \in \mathbb{R}^{2}$, for $(t, \theta) \in \mathbb{R} \times[0, \pi)$, and is perpendicular to the unit vector $\mathbf{n}_{\theta}=(\cos (\theta), \sin (\theta))$. Then, every straight line $\ell \subset \mathbb{R}^{2}$ can uniquely be represented by one $\ell_{t, \theta}$ with parameters $(t, \theta) \in \mathbb{R} \times[0, \pi)$. This allows us to reformulate the basic reconstruction problem accordingly.

To this end, we regard for $f \in \mathrm{L}^{1}\left(\mathbb{R}^{2}\right)$ its Radon transform

$$
\mathcal{R} f(t, \theta)=\int_{\ell_{t, \theta}} f(x, y) \mathrm{d} x \mathrm{~d} y \quad \text { for }(t, \theta) \in \mathbb{R} \times[0, \pi) .
$$

Note that the Radon transform $\mathcal{R}$ maps a bivariate function $f \equiv f(x, y)$ in Cartesian coordinates onto a bivariate function $\mathcal{R} f \equiv \mathcal{R} f(t, \theta)$ in polar coordinates, where $\mathcal{R} f(t, \theta)$ represents the line integral of $f$ over $\ell_{t, \theta}$.
Therefore, the basic reconstruction problem seeks for the inversion of the Radon transform $\mathcal{R}$ from Radon data

$$
\{\mathcal{R} f(t, \theta) \mid t \in \mathbb{R}, \theta \in[0, \pi)\} .
$$

For a comprehensive mathematical treatment of the Radon transform and its inversion, we refer to the textbooks [2], [7].

The outline of this paper is as follows. In Section II we describe the inversion of the Radon transform by the standard FBP formula. Moreover, we explain how the FBP can be stabilized by using a suitable low-pass filter with finite bandwidth. This stabilization modifies the FBP formula and so leads us to an approximate reconstruction formula, whose approximation quality depends on the choice of the low-pass filter. The objective of this paper is to analyse the approximation error occurring due to the use of a low-pass filter. Therefore, in Section III we prove $\mathrm{L}^{2}$ error estimates for the approximate FBP formula on Sobolev spaces of fractional order. For the purpose of illustration, numerical simulations are finally provided in Section IV.

\section{Filtered BACK Projection}

The inversion of the Radon transform $\mathcal{R}$ is well understood. It involves the (continuous) Fourier transform, here taken as

$$
\mathcal{F} g(S, \theta)=\int_{\mathbb{R}} g(t, \theta) \mathrm{e}^{-\mathrm{i} t S} \mathrm{~d} t \quad \text { for } \theta \in[0, \pi)
$$

for $g \equiv g(t, \theta)$ in polar coordinates satisfying $g(\cdot, \theta) \in \mathrm{L}^{1}(\mathbb{R})$ for all $\theta \in[0, \pi)$. Later in this work, we will also work with the Fourier transform on $\mathbb{R}^{2}$, defined as

$$
\mathcal{F} f\left(\omega_{1}, \omega_{2}\right)=\int_{\mathbb{R}^{2}} f(x, y) \mathrm{e}^{-\mathrm{i}\left(\omega_{1} x+\omega_{2} y\right)} \mathrm{d} x \mathrm{~d} y
$$

for $f \equiv f(x, y)$ in Cartesian coordinates, where $f \in \mathrm{L}^{1}\left(\mathbb{R}^{2}\right)$.

Another operator in the inversion of $\mathcal{R}$ is the back projection

$$
\mathcal{B} h(x, y)=\frac{1}{\pi} \int_{0}^{\pi} h(x \cos (\theta)+y \sin (\theta), \theta) \mathrm{d} \theta
$$

for $h \in \mathrm{L}^{1}(\mathbb{R} \times[0, \pi))$. Note that the back projection $\mathcal{B}$ maps a bivariate function $h \equiv h(t, \theta)$ in polar coordinates onto a bivariate function $\mathcal{B} h \equiv \mathcal{B} h(x, y)$ in Cartesian coordinates.

Now the inversion of the Radon transform is given by the filtered back projection formula (see e.g. [1, Theorem 6.2.])

$$
f(x, y)=\frac{1}{2} \mathcal{B}\left[\mathcal{F}^{-1}(|S| \mathcal{F}(\mathcal{R} f)(S, \theta))\right](x, y)
$$

which holds for any function $f \in \mathrm{L}^{1}\left(\mathbb{R}^{2}\right) \cap \mathscr{C}\left(\mathbb{R}^{2}\right)$ satisfying $\mathcal{R} f(\cdot, \theta) \in \mathrm{L}^{1}(\mathbb{R})$ for all $\theta \in[0, \pi)$. 
We remark that the FBP formula is numerically unstable. Indeed, by the application of the filter $|S|$ to the Fourier transform $\mathcal{F}(\mathcal{R} f)$ in (3), high frequency components of $\mathcal{R} f$ are amplified by the magnitude of $|S|$. Therefore, the filtered back projection formula is in particular highly sensitive with respect to noise. Needless to say that this is critical in many relevant application scenarios, where a reconstruction by FBP would lead to an undesired corruption of the image.

In order to reduce the sensitivity of the FBP formula with respect to noise, the filter $|S|$ in (3) is replaced by a low-pass filter $A_{L}(S)$ of bandwidth $L>0$. Commonly used low-pass filters have the form $A_{L}(S)=|S| W_{L}(S)$, where $W_{L}(S)=$ $W\left(\frac{S}{L}\right)$ for a suitable window function $W: \mathbb{R} \longrightarrow \mathbb{R}$ satisfying the following two properties.

- $W: \mathbb{R} \longrightarrow \mathbb{R}$ is even, i.e., $W(S)=W(-S)$ for all $S$;

- $W$ is compactly supported with $\operatorname{supp}(W) \subseteq[-1,1]$, i.e., $W(S)=0$ for all $|S|>1$.

By the above two properties, $W_{L}$ is also even with compact support contained in $[-L, L]$, i.e., $\operatorname{supp}\left(W_{L}\right) \subseteq[-L, L]$. In particular, $W_{L} \in \mathrm{L}^{1}(\mathbb{R})$, and so any low-pass filter of the form $A_{L}(S)=|S| W_{L}(S)$ is in $\mathrm{L}^{1}(\mathbb{R})$, in contrast to $|S|$.

Next, we replace the filter $|S|$ in (3) by a low-pass filter $A_{L}$, in which case the reconstruction of $f$ is no longer exact. With assuming $A_{L} \in \mathrm{L}^{1}(\mathbb{R})$, we can simplify the resulting approximate FBP formula as

$$
\begin{aligned}
f_{L}(x, y) & :=\frac{1}{2} \mathcal{B}\left(\mathcal{F}^{-1}\left[A_{L}(S) \mathcal{F}(\mathcal{R} f)(S, \theta)\right]\right)(x, y) \\
& =\frac{1}{2} \mathcal{B}\left(\mathcal{F}^{-1} A_{L} * \mathcal{R} f\right)(x, y)
\end{aligned}
$$

where $*$ denotes the usual convolution product. We can further simply this representation of $f_{L}$ by involving the band-limited function

$$
q_{L}(t, \theta)=\mathcal{F}^{-1} A_{L}(t) \quad \text { for }(t, \theta) \in \mathbb{R} \times[0, \pi),
$$

in which case we get

$$
f_{L}=\frac{1}{2} \mathcal{B}\left(q_{L} * \mathcal{R} f\right) .
$$

For the sake of brevity, we call any application of the approximate FBP formula (4) an FBP method. Therefore, each FBP method provides one approximation $f_{L}$ to $f, f_{L} \approx f$, whose quality depends on the choice of the low-pass filter $A_{L}$. In the following, we analyse the intrinsic error of the FBP method, which is incurred by the chosen low-pass filter $A_{L}$, i.e., we wish to analyse the error

$$
e_{L}=f-f_{L} .
$$

We remark that pointwise and $\mathrm{L}^{\infty}$ error estimates on $e_{L}$ were proven by Munshi et al. in [4]. Their results are further supported by numerical experiments in [5]. Error bounds on the $\mathrm{L}^{p}$-norm of $e_{L}$, in terms of an $\mathrm{L}^{p}$-modulus of continuity of $f$, were proven by Madych in [3]. In the following section, we prove $\mathrm{L}^{2}$ error estimates on $e_{L}$, where our approach involves Sobolev spaces of fractional order and so our approach is essentially different from that of Madych in [3].
Before doing so, it is convenient to rewrite the approximate reconstruction formula (4) in terms of the target function $f$. To be more precise, we seek for a function $K_{L}: \mathbb{R}^{2} \longrightarrow \mathbb{R}$ satisfying

$$
f_{L}=f * K_{L}
$$

This relation will play a central role in the error analysis of Section IIII The construction of such a function $K_{L}$ is based on the following useful relation between the convolution product, the back projection operator and the Radon transform, see [7]. Theorem II.1.3].

Lemma 1: Let $f \equiv f(x, y) \in \mathrm{L}^{1}\left(\mathbb{R}^{2}\right)$ be a bivariate function in Cartesian coordinates and $g \equiv g(t, \theta)$ be a bivariate function in polar coordinates with $g(\cdot, \theta) \in \mathrm{L}^{1}(\mathbb{R})$ for all $\theta \in[0, \pi)$. Then, the identity

$$
(\mathcal{B} g * f)(X, Y)=\mathcal{B}(g * \mathcal{R} f)(X, Y)
$$

holds for all $(X, Y) \in \mathbb{R}^{2}$.

Proof: For $(X, Y) \in \mathbb{R}^{2}$, we have

$(\mathcal{B} g * f)(X, Y)=\int_{\mathbb{R}} \int_{\mathbb{R}} \mathcal{B} g(X-x, Y-y) \cdot f(x, y) \mathrm{d} x \mathrm{~d} y$,

where, with letting $\bar{x}=X-x$ and $\bar{y}=Y-y$,

$$
\mathcal{B} g(\bar{x}, \bar{y})=\frac{1}{\pi} \int_{0}^{\pi} g(\bar{x} \cos (\theta)+\bar{y} \sin (\theta), \theta) \mathrm{d} \theta .
$$

By the substitutions

$$
\begin{aligned}
& x=t \cos (\theta)-s \sin (\theta) \\
& y=t \sin (\theta)+s \cos (\theta)
\end{aligned}
$$

we get $\mathrm{d} x \mathrm{~d} y=\mathrm{d} s \mathrm{~d} t$ and, therefore, with

$\mathcal{R} f(t, \theta)=\int_{\mathbb{R}} f(t \cos (\theta)-s \sin (\theta), t \sin (\theta)+s \cos (\theta)) \mathrm{d} s$ and $s_{\theta}=X \cos (\theta)+Y \sin (\theta)$, we can conclude

$$
\begin{aligned}
(\mathcal{B} g * f)(X, Y) & =\frac{1}{\pi} \int_{0}^{\pi} \int_{\mathbb{R}} g\left(s_{\theta}-t, \theta\right) \cdot \mathcal{R} f(t, \theta) \mathrm{d} t \mathrm{~d} \theta \\
& =\frac{1}{\pi} \int_{0}^{\pi}(g * \mathcal{R} f)\left(s_{\theta}, \theta\right) \mathrm{d} \theta \\
& =\mathcal{B}(g * \mathcal{R} f)(X, Y),
\end{aligned}
$$

as stated.

Consequently, for $q_{L} \in \mathrm{L}^{1}(\mathbb{R} \times[0, \pi))$, we define the convolution kernel $K_{L}: \mathbb{R}^{2} \longrightarrow \mathbb{R}$ by

$$
K_{L}(x, y):=\frac{1}{2} \mathcal{B} q_{L}(x, y) \quad \text { for }(x, y) \in \mathbb{R}^{2} .
$$

By using Lemma 1 we obtain the desired representation for the reconstruction $f_{L}$ by

$$
f_{L}(x, y)=\frac{1}{2} \mathcal{B}\left(q_{L} * \mathcal{R} f\right)(x, y)=\left(f * K_{L}\right)(x, y)
$$

for all $(x, y) \in \mathbb{R}^{2}$. 


\section{ERROR ESTIMATES}

In this section, we prove an $\mathrm{L}^{2}$ estimate for the error in (5), where our upper bound on the $\mathrm{L}^{2}$-norm of $e_{L}$ will be split into one term depending on the window function $W$ and another one depending on its bandwidth $L>0$.

Before we turn to our error analysis, we wish to first discuss the special case of the Ram-Lak filter, given as

$$
A_{L}(S)=\left\{\begin{array}{cl}
|S| & \text { for } S \in[-L, L] \\
0 & \text { otherwise. }
\end{array}\right.
$$

Note that the Ram-Lak filter's window function $W$ is given by the characteristic function $\chi_{[-1,1]}$ of the interval $[-1,1]$, so that $W_{L} \equiv \chi_{[-L, L]}$. From this observation, we see that the reconstruction error $e_{L}$ vanishes identically, $e_{L} \equiv 0$, for functions $f$ with band-limited Radon transform $\mathcal{R} f$, provided that the bandwidth $L$ is at least as large as the largest frequency contained in $\mathcal{R} f$. Indeed, in this case the Ram-Lak FBP formula (4) coincides with the FBP formula (3), so that $f \equiv f_{L}$. Yet it remains to discuss how reasonable it is to assume for $f$ a band-limited Radon transform $\mathcal{R} f$.

To further elaborate this, let us recall the central slice theorem (see e.g. [1. Theorem 6.1.]), which states that for any $f \in \mathrm{L}^{1}\left(\mathbb{R}^{2}\right) \cap \mathscr{C}\left(\mathbb{R}^{2}\right)$ the identity

$$
\mathcal{F} f(S \cos (\theta), S \sin (\theta))=\mathcal{F}(\mathcal{R} f)(S, \theta)
$$

holds for all $(S, \theta) \in \mathbb{R} \times[0, \pi)$. Therefore, the (bivariate) Fourier transform $\mathcal{F} f$ of $f \equiv f(x, y)$ is entirely determined by the (univariate) Fourier transforms of $\mathcal{R} f \equiv(\mathcal{R} f)(t, \theta)$.

Now $\mathcal{R} f$ is band-limited, iff $\mathcal{R} f$ has a compactly supported Fourier transform. However, for applications of medical image reconstruction, it is usually assumed that $f \not \equiv 0$ is compactly supported. But in this case, $\mathcal{R} f$ is compactly supported, too, and so the Fourier transform $\mathcal{F}(\mathcal{R} f)$ of $\mathcal{R} f \not \equiv 0$ cannot have compact support. The latter is due to the Paley-Wiener theorem (see e.g. [8, Theorem 7.22]), which states that the Fourier transform of a compactly supported function is analytic.

To conclude our discussion on the special case of the RamLak filter, we see that, for compactly supported $f \not \equiv 0$, the error $e_{L}$ of the Ram-Lak FBP method cannot be zero for finite bandwidth $L>0$. But if we let $L$ tend to infinity, the Ram-Lak FBP method will, in the limit, coincide with the filtered back projection formula (3), so that the error vanishes, $e_{\infty} \equiv 0$.

Let us now turn to the analysis of the $\mathrm{L}^{2}$ reconstruction error. To this end, we assume $f \in \mathrm{L}^{1}\left(\mathbb{R}^{2}\right) \cap \mathrm{L}^{2}\left(\mathbb{R}^{2}\right)$, in which case we get

$$
\begin{aligned}
\left\|f-f_{L}\right\|_{\mathrm{L}^{2}\left(\mathbb{R}^{2}\right)}^{2} & =\left\|f-f * K_{L}\right\|_{\mathrm{L}^{2}\left(\mathbb{R}^{2}\right)}^{2} \\
& =\frac{1}{2 \pi}\left\|\mathcal{F} f-\mathcal{F} f \cdot \mathcal{F} K_{L}\right\|_{\mathrm{L}^{2}\left(\mathbb{R}^{2} ; \mathbb{C}\right)}^{2}
\end{aligned}
$$

by using the Rayleigh-Plancherel theorem.

By letting $W_{L}(x, y):=W_{L}(r)$, for $r=\sqrt{x^{2}+y^{2}}$ and $(x, y) \in \mathbb{R}^{2}$, we obtain a radially symmetric bivariate function $W_{L}: \mathbb{R}^{2} \rightarrow \mathbb{R}$. It can be verified that this radial window $W_{L}$ is given by the Fourier transform of the convolution kernel $K_{L}$ in (6), i.e., for $K_{L} \in \mathrm{L}^{1}\left(\mathbb{R}^{2}\right)$ we can establish the identity

$$
W_{L}(x, y)=\mathcal{F} K_{L}(x, y) \quad \text { for all }(x, y) \in \mathbb{R}^{2},
$$

in consequence of [7, Theorem II.1.4]. Hence, it follows that

$$
\begin{aligned}
\left\|e_{L}\right\|_{\mathrm{L}^{2}\left(\mathbb{R}^{2}\right)}^{2} & =\frac{1}{2 \pi}\left\|\mathcal{F} f-W_{L} \cdot \mathcal{F} f\right\|_{\mathrm{L}^{2}\left(\mathbb{R}^{2} ; \mathbb{C}\right)}^{2} \\
& =\frac{1}{2 \pi} \int_{\mathbb{R}} \int_{\mathbb{R}}\left|\left(\mathcal{F} f-W_{L} \cdot \mathcal{F} f\right)(x, y)\right|^{2} \mathrm{~d} x \mathrm{~d} y .
\end{aligned}
$$

To continue our analysis, we split the above representation of the $L^{2}$-error $\left\|e_{L}\right\|_{L^{2}\left(\mathbb{R}^{2}\right)}$ into a sum of two integrals,

$$
\left\|e_{L}\right\|_{\mathrm{L}^{2}\left(\mathbb{R}^{2}\right)}^{2}=I_{1}+I_{2},
$$

where

$$
\begin{aligned}
& I_{1}=\frac{1}{2 \pi} \int_{\left\|\left(\begin{array}{l}
x \\
y
\end{array}\right)\right\|_{2} \leq L}\left|\left(\mathcal{F} f-W_{L} \cdot \mathcal{F} f\right)(x, y)\right|^{2} \mathrm{~d} x \mathrm{~d} y \\
& I_{2}=\frac{1}{2 \pi} \int_{\left\|\left(\begin{array}{l}
x \\
y
\end{array}\right)\right\|_{2}>L}|\mathcal{F} f(x, y)|^{2} \mathrm{~d} x \mathrm{~d} y .
\end{aligned}
$$

To further analyse the error terms in $I_{1}$ and $I_{2}$, we first need to introduce Sobolev spaces of fractional order. The Sobolev space $\mathrm{H}^{\alpha}\left(\mathbb{R}^{2}\right)$ of order $\alpha \in \mathbb{R}$ is defined as

$$
\mathrm{H}^{\alpha}\left(\mathbb{R}^{2}\right)=\left\{f \in \mathcal{S}^{\prime}\left(\mathbb{R}^{2}\right) \mid\|f\|_{\mathrm{H}^{\alpha}\left(\mathbb{R}^{2}\right)}<\infty\right\},
$$

and equipped with the norm $\|\cdot\|_{\mathrm{H}^{\alpha}\left(\mathbb{R}^{2}\right)}$, given by

$$
\|f\|_{\mathrm{H}^{\alpha}\left(\mathbb{R}^{2}\right)}^{2}=\int_{\mathbb{R}^{2}}\left(1+\|\omega\|_{2}^{2}\right)^{\alpha}|\mathcal{F} f(\omega)|^{2} \mathrm{~d} \omega,
$$

where $\mathcal{S}^{\prime}$ denotes the space of tempered distributions. It can be shown that for any non-negative integer $\alpha \in \mathbb{N}_{0}$ the space $\mathrm{H}^{\alpha}\left(\mathbb{R}^{2}\right)$ consists of all functions whose (distributional) derivatives up to order $\alpha$ are square-integrable.

In relevant application of (medical) image processing, Sobolev spaces of compactly supported functions,

$$
\mathrm{H}_{0}^{\alpha}(\Omega)=\left\{f \in \mathrm{H}^{\alpha}\left(\mathbb{R}^{2}\right) \mid \operatorname{supp}(f) \subseteq \bar{\Omega}\right\},
$$

on open and bounded domain $\Omega \subset \mathbb{R}^{2}$, and of fractional order $\alpha$ play an important role (cf. [6]). In fact, a density function $f$ of an image in $\Omega \subset \mathbb{R}^{2}$ has usually jumps along smooth curves, but is otherwise smooth off these curve singularities. Such functions belong to a Sobolev space $\mathrm{H}_{0}^{\alpha}\left(\mathbb{R}^{2}\right)$ for $\alpha<\frac{1}{2}$. Thus, we can describe the density of an image as a function in a Sobolev space $\mathrm{H}_{0}^{\alpha}(\Omega)$ whose order $\alpha$ is close to $\frac{1}{2}$.

Now let us return to the required $\mathrm{L}^{2}$ error estimates for $e_{L}$. For $f \in \mathrm{H}^{\alpha}\left(\mathbb{R}^{2}\right)$, with $\alpha \geq 0$, the integral $I_{2}$ can be bounded above by

$$
\begin{aligned}
I_{2} & =\frac{1}{2 \pi} \int_{r>L}\left(1+r^{2}\right)^{\alpha}\left(1+r^{2}\right)^{-\alpha}|\mathcal{F} f(x, y)|^{2} \mathrm{~d} x \mathrm{~d} y \\
& \leq \frac{1}{2 \pi} \int_{r>L}\left(1+r^{2}\right)^{\alpha} L^{-2 \alpha}|\mathcal{F} f(x, y)|^{2} \mathrm{~d} x \mathrm{~d} y \\
& \leq \frac{1}{2 \pi} L^{-2 \alpha}\|f\|_{\mathrm{H}^{\alpha}\left(\mathbb{R}^{2}\right)}^{2},
\end{aligned}
$$

where we let $r=\sqrt{x^{2}+y^{2}}$. 
As regards integral $I_{1}$, we consider a fixed low-pass filter $A_{L}(S)=|S| W_{L}(S)$ with window function $W \in \mathrm{L}^{\infty}(\mathbb{R})$. Then, for $f \in \mathrm{L}^{1}\left(\mathbb{R}^{2}\right) \cap \mathrm{L}^{2}\left(\mathbb{R}^{2}\right)$, the integral $I_{1}$ can be bounded above by

$$
I_{1} \leq\|1-W\|_{\infty,[-1,1]}^{2}\|f\|_{\mathrm{L}^{2}\left(\mathbb{R}^{2}\right)}^{2},
$$

since $\left\|1-W_{L}\right\|_{\infty,[-L, L]}=\|1-W\|_{\infty,[-1,1]}$.

We can summarize the discussion of this section as follows.

Theorem 1 ( $\mathrm{L}^{2}$-error estimate): Let $f \in \mathrm{L}^{1}\left(\mathbb{R}^{2}\right) \cap \mathrm{H}^{\alpha}\left(\mathbb{R}^{2}\right)$ for some $\alpha \geq 0$, let $W \in \mathrm{L}^{\infty}(\mathbb{R})$ and $K_{L} \in \mathrm{L}^{1}\left(\mathbb{R}^{2}\right)$. Then, the $\mathrm{L}^{2}$-norm of the FBP reconstruction error $e_{L}=f-f_{L}$ in (5) can be bounded above by

$$
\left\|e_{L}\right\|_{L^{2}\left(\mathbb{R}^{2}\right)} \leq\|1-W\|_{\infty,[-1,1]}\|f\|_{L^{2}\left(\mathbb{R}^{2}\right)}+L^{-\alpha}\|f\|_{\alpha},
$$

where we let

$$
\|f\|_{\alpha}^{2}=\frac{1}{2 \pi}\|f\|_{\mathrm{H}^{\alpha}\left(\mathbb{R}^{2}\right)}^{2}
$$

We can conclude from the above theorem that the choice of both the window function $W$ and the bandwidth $L$ are of fundamental importance for the $\mathrm{L}^{2}$-error of the FBP method. In fact, the error term $\|1-W\|_{\infty,[-1,1]}$ can be used to evaluate the quality of the window function $W$. Note that the window function $W \equiv \chi_{[-1,1]}$ of the Ram-Lak filter is the unique minimizer of that quality indicator, and so the Ram-Lak filter is in this sense the optimal low-pass filter.

Moreover, note that the smoothness of $f$ determines the decay rate of the other error term by

$$
L^{-\alpha}\|f\|_{\alpha}=\mathcal{O}\left(L^{-\alpha}\right) \quad \text { for } L \rightarrow \infty .
$$

But the right hand side of our $\mathrm{L}^{2}$ error estimate can only tend to 0 , iff we choose the Ram-Lak filter with window $W=\chi_{[-1,1]}$ and let $L$ tend to $\infty$. This observation complies with the derived conditions under which we achieve an exact reconstruction of the target function $f$ by the FBP formula (3), as this is explained at the outset of this section.

Finally, note that, for fixed target function $f$ and bandwidth $L$, the obtained error estimate is affine-linear with respect to the $L^{\infty}$ distance between the window function and the constant function 1 . This relation will also be observed numerically in the following section.

\section{NuMERICAL RESUlts}

In this section, we provide selected numerical examples to evaluate the FBP reconstruction error numerically and to validate our $\mathrm{L}^{2}$ error estimate from the previous section.

Note that the FBP reconstruction formula assume the Radon data $\mathcal{R} f(t, \theta)$ to be available for all $t \in \mathbb{R}$ and $\theta \in[0, \pi)$. In practice, however, only a finite number of Radon values are given,

$$
(\mathcal{R} f)_{j, k}=\mathcal{R} f\left(t_{j}, \theta_{k}\right)
$$

for $-M \leq j \leq M$ and $0 \leq k \leq N-1$ for some $M, N \in \mathbb{N}$. To reconstruct $f$ from given Radon data (8), this requires a suitable discretization of the FBP method. But this will lead

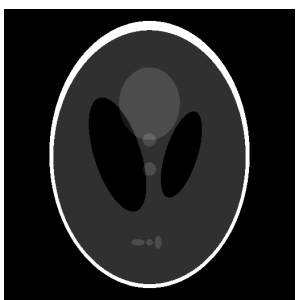

(a) Phantom

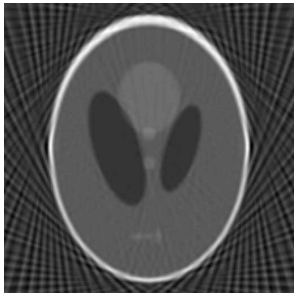

(b) Reconstruction

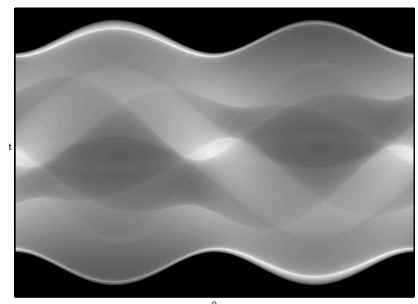

(c) Sinogram

Fig. 1. Reconstruction of the Shepp-Logan phantom

to discretization errors, which are not covered by our error analysis and cannot be avoided.

To implement the approximate reconstruction formula

$$
f_{L}=\frac{1}{2} \mathcal{B}\left(\mathcal{F}^{-1} A_{L} * \mathcal{R} f\right),
$$

we need to discretize the back projection $\mathcal{B}$, the inverse Fourier transform $\mathcal{F}^{-1}$, the convolution product $*$ and the Radon transform $\mathcal{R}$. For the sake of brevity, we do not intend to explain details concerning the discretization. For our purposes it is sufficient to say that we use parallel beam geometry for the discretization of the Radon transform and otherwise refer to the textbook [1] for further details.

In our numerical experiments, we considered using the popular Shepp-Logan phantom as a test function, whose Radon transform can be calculated analytically. The Shepp-Logan phantom consists of ten ellipses of constant densities, but different sizes, eccentricities and locations, see Fig. 11a). The phantom was introduced by Shepp and Logan [9] and it simulates a cross-section of the human head. In our version, we modified the densities of the different ellipses from the original Shepp-Logan phantom in order to get a higher contrast in the image for a better visual perception. Fig. 11(c) shows the corresponding sinogram, i.e., the Radon data of the phantom in the $(t, \theta)$-plane. The FBP reconstruction based on this test case is displayed in Fig. 1 (b), where we used the Ram-Lak filter with window $W=\chi_{[-1,1]}$, bandwidth $L=20$ and $N=45$ for the number of angles $\theta_{k}$.

To measure the reconstruction error, we used the standard root mean square error (RMSE), which is defined as

$$
\mathrm{RMSE}=\sqrt{\frac{1}{N \times M} \sum_{n=1}^{N} \sum_{m=1}^{M}\left(f_{n, m}-\left(f_{L}\right)_{n, m}\right)^{2}},
$$

where $N \times M$ is the total number of pixels in the images. 
For the numerical evaluation of the FBP method (4), the intrinsic error of the reconstruction, being incurred by the choice of the low-pass filter $A_{L}$, is of primary interest. However, we also need to take into account inevitable discretization errors, which are not covered by our error analysis.

Recall that our $\mathrm{L}^{2}$ error estimate states that for any function $f \in \mathrm{L}^{1}\left(\mathbb{R}^{2}\right) \cap \mathrm{H}^{\alpha}\left(\mathbb{R}^{2}\right)$ with smoothness $\alpha \geq 0$ we have

$$
\left\|f-f_{L}\right\|_{L^{2}\left(\mathbb{R}^{2}\right)} \leq\|1-W\|_{\infty,[-1,1]}\|f\|_{L^{2}\left(\mathbb{R}^{2}\right)}+L^{-\alpha}\|f\|_{\alpha} .
$$

Here, we see that, for fixed function $f$ and bandwidth $L$, the performance of the used low-pass filter $A_{L}$ is governed by the $\mathrm{L}^{\infty}$ distance between the window function $W$ and the constant function 1. Moreover, the error formula predicts an affine-linear behaviour of the error $e_{L}$ with respect to $\|1-W\|_{\infty,[-1,1]}$.

To investigate the error $e_{L}$ numerically, we have employed three commonly used low-pass filters:

\begin{tabular}{l|c|c} 
Name & $W(R)$ for $|R| \leq 1$ & $\|1-W\|_{\infty,[-1,1]}$ \\
\hline Ram-Lak & 1 & 0 \\
Shepp-Logan & $\operatorname{sinc}\left(\frac{\pi R}{2}\right)$ & $1-\frac{2}{\pi}$ \\
Hamming & $\beta+(1-\beta) \cos (\pi R)$ & $2(1-\beta)$
\end{tabular}

In addition to the popular Ram-Lak and Shepp-Logan filter, we considered using the Hamming filter for different choices of parameters $\beta \in\left[\frac{1}{2}, 1\right]$. This decision was taken in order to work with a sequence of filters of the same form, but with different values for $\|1-W\|_{\infty,[-1,1]}$. For the Hamming filter, we see that the quantity $\|1-W\|_{\infty,[-1,1]}$ decreases for $\beta$ increasing. Hence, for the Hamming filter, the reconstruction error should increase for decreasing $\beta$.

Fig. 2 shows, for the test case of the Shepp-Logan phantom, the RMSE as a function of $\|1-W\|_{\infty,[-1,1]}$ for different choices of bandwidth $L$ and number of views $N$.

We can describe our numerical results as follows.

First of all, we have observed an increasing RMSE at increasing $\|1-W\|_{\infty,[-1,1]}$ in all of our numerical experiments. This is exactly the behaviour we expected, due to the first term in our $\mathrm{L}^{2}$ error estimate, given as

$$
\|1-W\|_{\infty,[-1,1]}\|f\|_{L^{2}\left(\mathbb{R}^{2}\right)} .
$$

Moreover, the affine-linear behaviour of the RMSE with respect to $\|1-W\|_{\infty,[-1,1]}$ is clearly visible (see Fig. 2).

Secondly, we see that the RMSE decreases at increasing bandwidth $L$. This behaviour complies with the second term in our $\mathrm{L}^{2}$ error estimate,

$$
L^{-\alpha}\|f\|_{\mathrm{H}^{\alpha}\left(\mathbb{R}^{2}\right)} \quad \text { for } \alpha \geq 0 .
$$

In particular, comparing our numerical results for an equal number $N=18$ of views but for different bandwidths, $L=6$ (Fig. 2(a)) and $L=10$ (Fig. 2 (b)), we see that the RMSE decreases, for all filters, as predicted by our error estimate.

When increasing the number of views from $N=18$ to $N=36$, at fixed bandwidth $L=10$, we see that the RMSE decreases further (see Fig. 2(b),(c)). We remark at

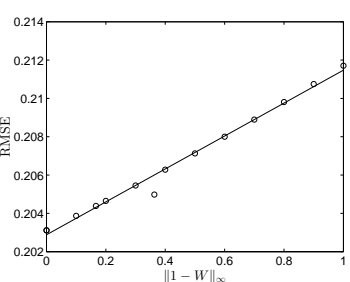

(a) $L=6, N=18$

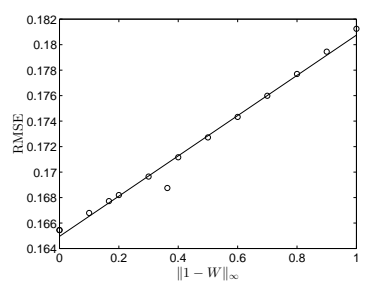

(c) $L=10, N=36$

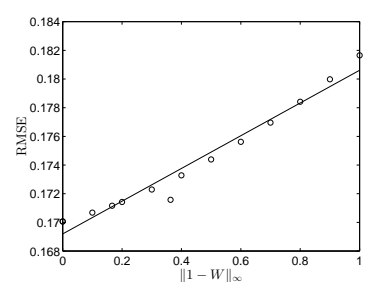

(b) $L=10, N=18$

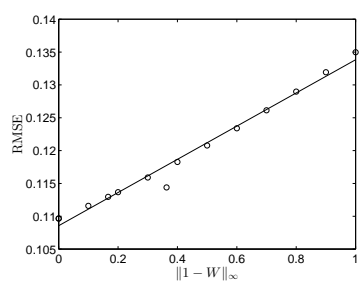

(d) $L=16, N=100$
Fig. 2. RMSE for the Shepp-Logan phantom

this point that the value of $N$ does not contribute to the intrinsic FBP reconstruction error, but it rather affects the discretization error: the larger $N$, the smaller the discretization error. Increasing $N$ is necessary for larger bandwidths $L$, since in that case the intrinsic error is small, so that a finer discretization can contribute more to the reduction of the RMSE (see Fig. 2(d)).

In conclusion, the numerical results of this section comply with the predictions from our theoretical $\mathrm{L}^{2}$ error estimates, although they do not cover the discretization errors. But the affine-linear behaviour of the error with respect to the magnitude of $\|1-W\|_{\infty,[-1,1]}$ is clearly observed.

\section{REFERENCES}

[1] T.G. Feeman: The Mathematics of Medical Imaging: A Beginner's Guide. Springer Undergraduate Texts in Mathematics and Technology (SUMAT), Springer, New York, 2010.

[2] S. Helgason: The Radon Transform. Progress in Mathematics, Volume 5, Birkhäuser, Boston, 2nd Edition (1999).

[3] W.R. Madych: Summability and Approximate Reconstruction from Radon Transform Data. Contemporary Mathematics, Volume 113, Amer. Math. Soc., Providence, 1990, 189-219.

[4] P. Munshi, R.K.S. Rathore, K.S. Ram, M.S. Kalra: Error estimates for tomographic inversion. Inverse Problems 7(3), 1991, 399-408.

[5] P. Munshi, R.K.S. Rathore, K.S. Ram, M.S. Kalra: Error analysis of tomographic filters II: results. NDT \& E International 26(5), 1993, 235240.

[6] F. Natterer: A Sobolev Space Analysis of Picture Reconstruction. SIAM Journal on Applied Mathematics 39(3), 1980, 402-411.

[7] F. Natterer: The Mathematics of Computerized Tomography. Classics in Applied Mathematics 32, SIAM, Philadelphia, 2001.

[8] W. Rudin: Functional Analysis. International Series in Pure and Applied Mathematics, McGraw-Hill, New-York, 2nd Edition (1991).

[9] L.A. Shepp, B.F. Logan: The Fourier Reconstruction of a Head Section. IEEE Transactions on Nuclear Science NS-21, 1974, 21-43. 